\title{
Estudio de supervivencia de tres sistemas de ataches extracoronarios utilizados
}

en prótesis mixta

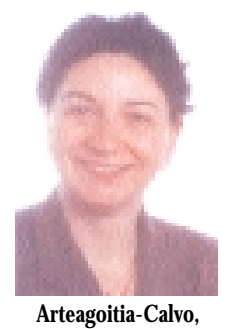

$\mathrm{M}^{\mathrm{a}}$ Iciar

\section{Survival study of three systems of extracoronary attachments used in mixed prostheses}

\section{Arteagoitia-Calvo, Ma Iciar* \\ Sabras- Puras, Fernando* \\ San Martín-Martínez, J. Antonio** \\ Gi|-Lozano, Jaime***}

* Profesor asociado de Prótesis Dental y Maxilofacial del Departamento de Estomatología. Universidad del País Vasco.

** Profesor titular de Prótesis Dental y Maxilofacial del Departamento de Estomatología. Universidad del País Vasco.

*** Catedrático de Prótesis Dental y Maxilofacial del Departamento de Estomatología. Universidad del País Vasco.

\section{Correspondencia}

Ma Iciar Arteagoitia Calvo

Departamento de Estomatología

Universidad del País Vasco

Barrio Sarriena s/n

Leioa 48940 - Lejona (Vizcaya)

E-mail: arteagoitia@infomed.es
Resumen. Introducción: El objetivo es determinar el comportamiento clínico y la supervivencia de tres sistemas de ataches extracoronarios: Roach, MiniDalbo y MiniSG. Material y método: Se realizó un estudio retrospectivo, de las historias clínicas de pacientes a los que como mínimo hacía 48 meses se habían colocado prótesis mixta con los citados ataches. Se registraron los aspectos clínicos que podían influir en la supervivencia: número de pilares ferulizados, extremo libre, maxilar y tipo de dentición del antagonista. El tamaño muestral incluyó 163 ataches. Se consideró evento terminal la fractura del atache, del pilar o el despegamiento de la corona. El estudio fue a tiempo completo. Resultados: La supervivencia media fue de 46 meses (IC 95\% 44,9-47,3 ). Se presentaron 24 complicaciones en 21 ataches. El atache MiniSG presenta el menor número de complicaciones $9 \%($ IC 95\%0-18,12 ). Ni el Test de Breslow, ni la regresión de Cox mostraron valores significativos cuando se contrastaron las curvas de supervivencia de las distintas variables estudiadas, incluido el tipo de atache. Conclusion: El atache MiniSG presenta mejor comportamiento clínico que el Roach y el MiniDalbo pero las diferencias no son estadísticamente significativas.

Palabras Clave: Prótesis mixta, Atache, Roach, MiniDalbo, MiniSG, Supervivencia.

Abstract: Introduction: The objective is to determine the clinical behaviour and survival of three systems of extra coronary attachments: Roach, MiniDalbo and MiniSG. Material and method: A retrospective study was performed on clinical histories of patients who had been treated with mixed prostheses using the attachments mentioned, at least 48 months prior to the study. Clinical aspects which might influence the survival were recorded: number of splinted abutment teeth, free-end situations, jaw involved and dentition type of opposing arch. The sample size included 163 attachments. Terminal event was attachmnt/teeth fracture or crown decementation. Results: Mean survival rate was 46 months (IC 95\% 44.9-47.3). 24 complications appeared in 21 attachments. The attachment MiniSG presents the fewest complications 9\% IC 95\% 018.12). Neither the Breslow Test nor Cox Regression showed significant values on contrasting the survival curves of the different variables studied, including attachment type. Conclusion: The attachment MiniSG presents better clinical behaviour than the Roach and the MiniDalbo, although the differences are not statistically significant.

Key Words: Mixed prosthesis, Attachment, Roach, Minidalbo, MiniSG, Survival.

\begin{tabular}{ccc}
\hline Fecha recepción & Fecha última revisión & Fecha aceptación \\
$29-11-2002$ & $5-02-2003$ & $25-02-2003$ \\
\hline
\end{tabular}

BIBUD [1138-123X (2003)8:3; mayo-junio 245-348]

Arteagoitia-Calvo MI, Sabras-Puras F, San Martín-Martínez J A, Gil-Lozano J. Estudio de supervivencia de tres sistemas de ataches extracoronarios utilizados en prótesis mixta. RCOE 2003;8(3):275-283. 


\section{Introducción}

A pesar de los avances quirúrgicos y protésicos para realizar una correcta rehabilitación oral, la prótesis mixta con ataches continúa siendo una de las alternativas de tratamiento, porque permite lograr una función y estética eficaz. Si bien son múltiples los tipos de ataches ${ }^{1}$ y algunos de ellos vienen siendo utilizados desde hace muchos años, es muy pobre la evidencia que existe sobre su comportamiento clínico.

Se han realizado estudios in vitro para tratar de determinar su comportamiento, su resistencia y como se realiza su distribución de fuerzas ${ }^{2-4}$. Asimismo, se han publicado estudios clínicos con algunos sistemas pero no se ha podido establecer cuales son los factores que influyen en la evolución de la prótesis mixta, ni que atache es el más eficaz en cada caso ${ }^{5,6^{*}, 7 *, 8^{* *}, 9}$. En los últimos años es muy poca la literatura científica que sobre este tema se ha publicado.

Uno de los ataches extracoronarios mas frecuentemente utilizado en España es el atache Roach. Otros sistemas de ataches, de implantación más reciente, como el atache MiniSG parecen tener unas características que le auguran un buen comportamiento clínico.

Nuestro objetivo, tras al menos 48 meses de seguimiento en una muestra de 163 ataches, ha sido determinar los factores que influyen en la evolución de la prótesis mixta, y establecer qué atache es el más eficaz,

Figura 1. Tres sistemas de ataches utilizados en prótesis mixta: Roach, MiniDalbo y MiniSG.
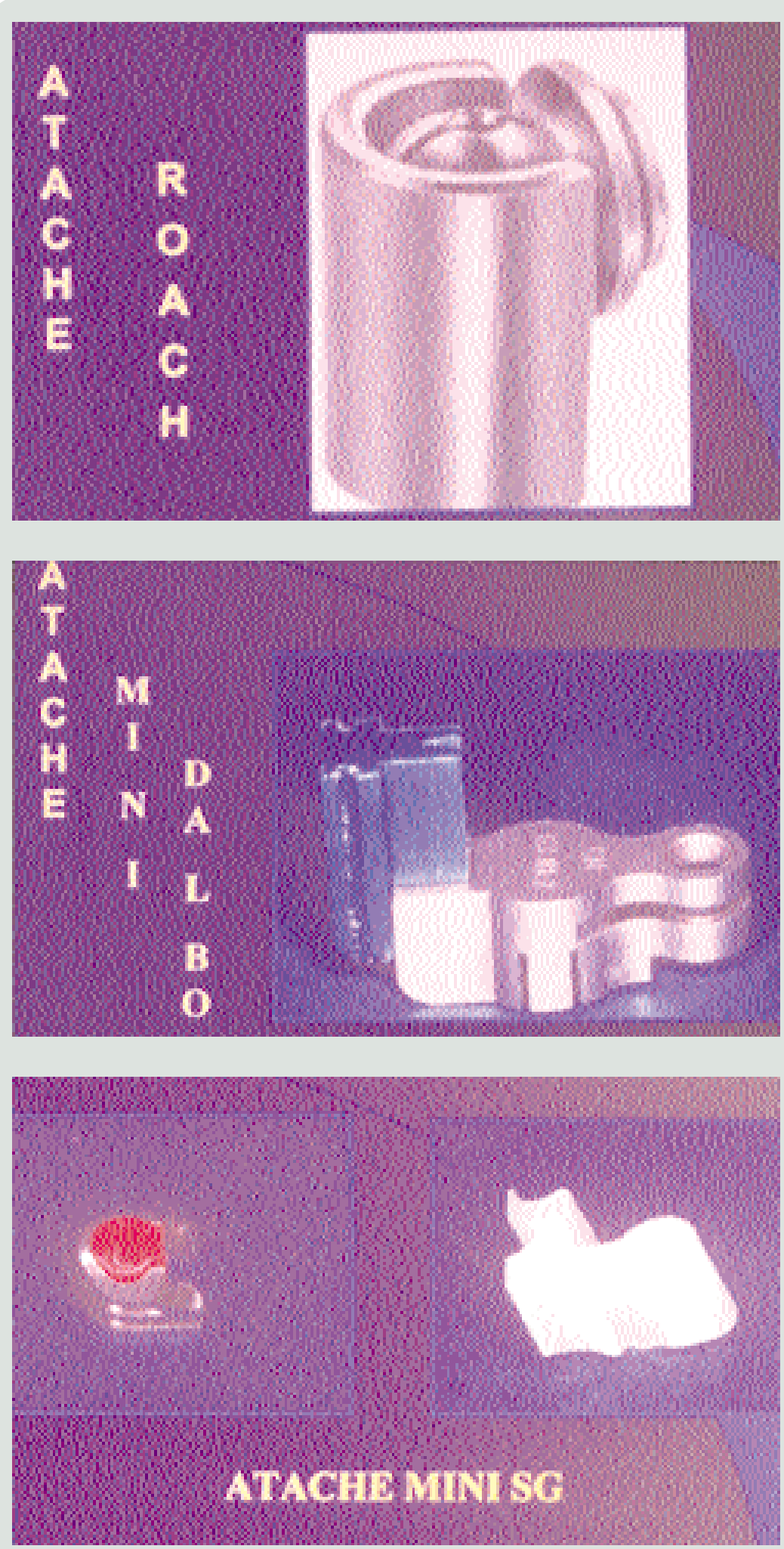

RCOE, 2003, Vol 8, №3, 275-283 

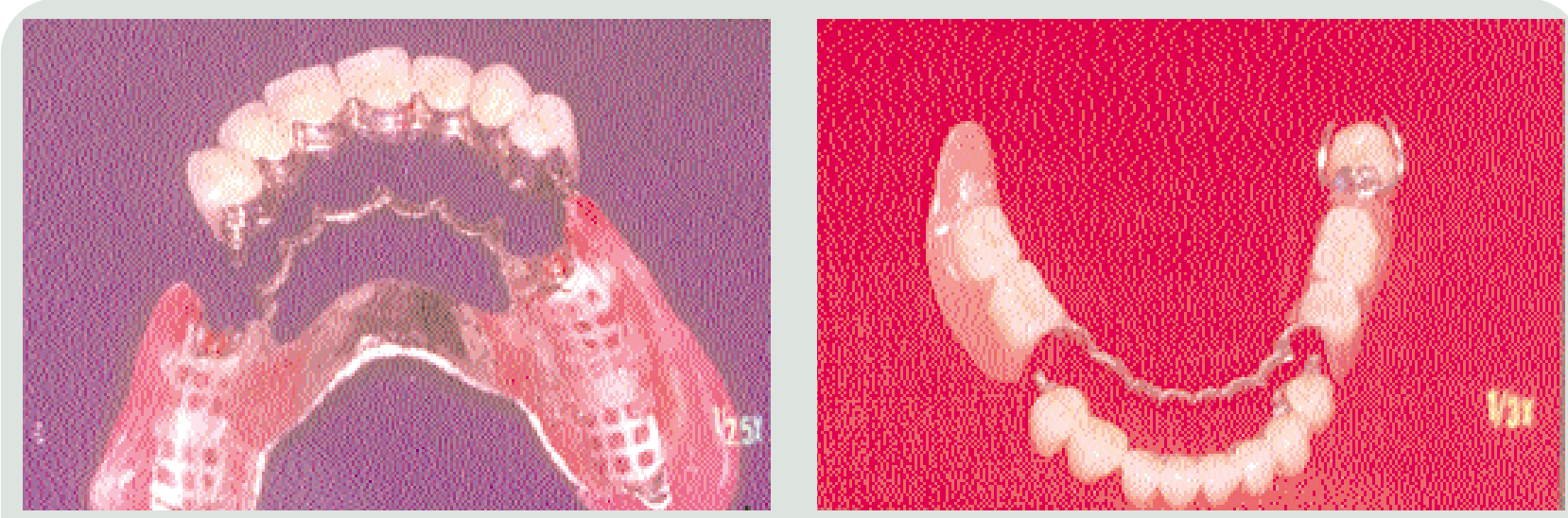

Figura 2. Diseño de las prótesis mixtas.

entendiendo como eficacia el menor número de complicaciones posibles y la mayor supervivencia.

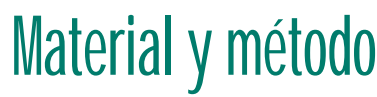

\section{Diseño del estudio}

Estudio observacional, analítico, retrospectivo, de cohortes históricas fijas de inicio. Análisis de supervivencia.

El estudio se ha realizado con las historias clínicas de los pacientes que habían sido atendidos por un único equipo profesional en una clínica de gestión privada.

Se realizó una revisión retrospectiva de las historias clínicas de los pacientes a los que se les habían colocado ataches como elemento de unión entre la prótesis fija y la prótesis removible. Se reclutaron de manera consecutiva todos los individuos de la población accesible que cumplían los siguientes criterios de inclusión:

- Ser portador de una prótesis mixta con ataches extracoronarios Roach, MiniDalbo y MiniSG fabricados por la misma casa comercial Cendre-\& Métaux $S A \circledast$, (Switzerland 8.2002) (fig. 1).
- El diseño de la prótesis y todos los ataches fueron realizados y colocados por el mismo profesional como mínimo 48 meses antes de comenzar el estudio.

- Los trabajos protésicos fueron realizados por el mismo laboratorio de prótesis.

- Los pilares no vitales habían sido restaurados con espiga-muñón colado.

- El cemento utilizado fue oxifosfato de zinc. (Flecks, Mizzy Inc (Cherry Hill,NJ 08002, USA)).

Ciento ochenta y cinco ataches cumplieron los criterios de inclusión. Excluimos del estudio a los pacientes bruxistas, con patología de la ATM, con implantes, con enfermedad periodontal (bolsas mayores de $5 \mathrm{~mm}$ y/o pérdida ósea mayor de $1 / 3$ ), pacientes psiquiátricos y un paciente que no estaba a riesgo constante a los dos años del tratamiento se sustituyó, en la arcada antagonista, una prótesis fija cerámica por una prótesis parcial removible con dientes de resina). Por estos motivos se excluyeron 21 pacientes, por lo que la muestra final consistió en 163 ataches.

\section{Diseño de las prótesis}

Prótesis fijas: los tallados se realizaron siguiendo los criterios protésicos de Shillinburg ${ }^{10}$. Todas las coronas ceramometálicas fueron confeccionadas con una estructura de metal a partir de la aleación metálica Option, y el recubrimiento estético de cerámica Vita ${ }^{\circledR}$. En las prótesis parciales removibles los conectores mayores en el maxilar superior fueron del tipo placas metálicas. En el maxilar inferior se diseñaron barras linguales 0 barras apoyadas en los cíngulos con microfresado de la prótesis fija. Las sillas o bases se confeccionaron con extensiones distales hasta el trígono retromolar en el maxilar inferior o incluyendo la tuberosidad en el superior. Todas las sillas fueron realizadas en acrílico para poder realizar rebases (fig. 2).

En los ataches las hembras eran de oro y los machos calcinables fueron colados en Option ${ }^{\circledR}$. Todos los trabajos se realizaron en articulador semiajustable Denar ${ }^{\circledR}$.

Hemos incluido a todos los pacientes en el estudio desde el mismo momento, en que se inserta la prótesis en la boca. El seguimiento en todos 
los casos ha sido completo (4 años), y ninguno de los pacientes se perdió en este tiempo. Los pacientes han estado a riesgo constante.

Todas las prótesis mixtas han sido revisadas una vez al año; exploración, radiografía y valoración de la necesidad de rebase en todos los casos por el mismo observador. Once revisiones se realizaron con un retraso superior a los 12 meses y en ningún caso superior a los 18.

\section{Protocolo de estudio}

Hemos registrado el tiempo y la edad, en años cumplidos, de la población de estudio.

1. Variables de estudio: hemos incluido aquellas variables que, en base a la literatura consultada, pueden influir en el comportamiento clínico de nuestras prótesis mixtas.

Tipo de atache: Roach 73, MiniDalbo 46 , MinisG 44.

Número de pilares: uno, dos, tres o más de tres pilares ferulizados.

Extremo libre: si o no.

Maxilar: superior o inferior.

Antagonista: prótesis removible, fija, dentición natural.

2. Variable resultado: (evento terminal). Se han incluido solamente aquellos accidentes que puedan de-terminarse por métodos objetivos de verificación: fractura de pilar, fractura del atache y despegamiento de la corona.

\section{Método estadístico}

Los datos obtenidos han sido estudiados mediante el Statistical Package for Social Science SPSS/PC. Hemos realizado:

1. Estudio descriptivo: variables cualitativas, frecuencias absolutas y relativas y para las variables cuantitati-

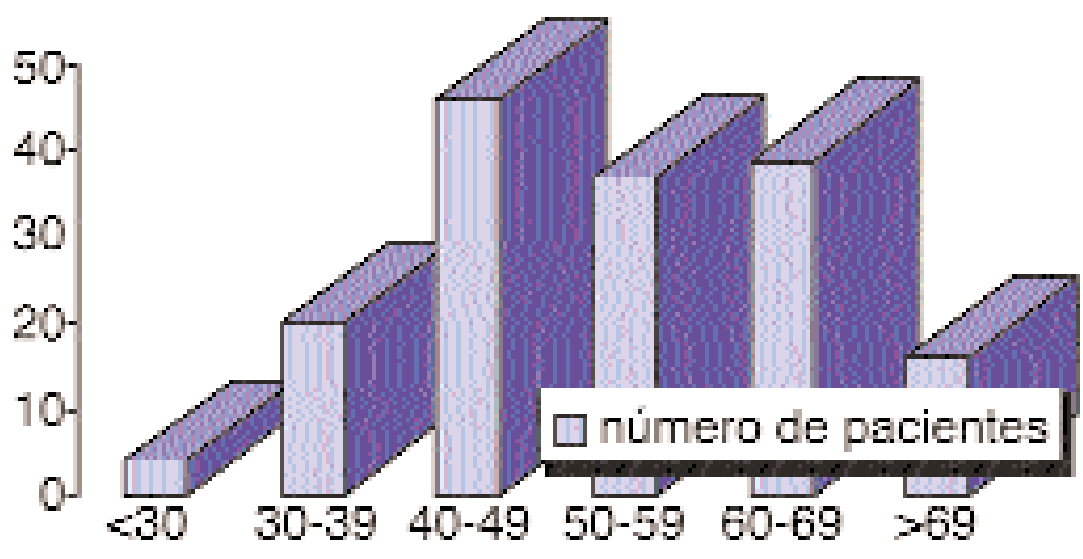

Figura 3. Histograma de la variable edad. La muestra está constituida fundamentalmente por pacientes entre 40 y 70 años.

\begin{tabular}{|c|c|c|c|c|c|c|c|}
\hline \multicolumn{8}{|c|}{ Tabla l: Características de las variables estudiadas } \\
\hline Tipo de atache & Roach & 73 & MiniDalbo & 46 & MinisG & 44 & \\
\hline $\begin{array}{l}\text { Número de pilares } \\
\text { ferulizados }\end{array}$ & 1 & 45 & 2 & 59 & 3 & 55 & 44 \\
\hline Extremo libre & $\mathrm{Si}$ & 97 & No & 66 & & & \\
\hline Arcada & Superior & 75 & Inferior & 88 & & & \\
\hline Antagonista & \begin{tabular}{|l|} 
Prótesis \\
removible
\end{tabular} & & Prótesis fija & & \begin{tabular}{|l|} 
Dientes \\
Naturales
\end{tabular} & 36 & \\
\hline
\end{tabular}

vas medidas basadas en momentos y en ordenaciones. Diagrama de cajas.

2. Estudio analítico mediante pruebas no paramétricas $U$ de ManWhitney y Kruskal Wallis.

3. Análisis de supervivencia de Kaplan Meier para predecir la probabilidad de ocurrencia y la evolución de la tasa de incidencia de los accidentes, tasa de riesgo a lo largo del seguimiento. Se ha utilizado el test de Breslow para contrastar si los diferentes niveles de un factor producen o no curvas de supervivencia significativamente distintas.

4. Estudio multivariante. Análisis de regresión de Cox.

El nivel de significación empleado en todos los análisis ha sido del $5 \%$ $\alpha=0,05$.

\section{Resultados}

\section{Estudio descriptivo}

La muestra la constituyeron 163 ataches, 129 colocados en mujeres y 29 en varones. La distribución por edades se presenta en la figura 3. Las características de las variables estudiadas se muestran en la tabla 1 . Las complicaciones a los cuatro años fueron 24 , y en tres casos se presentaron dos complicaciones, siendo la segunda en todos los casos el despegamiento. La complicación mas frecuente fue el despegamiento. La distribu- 


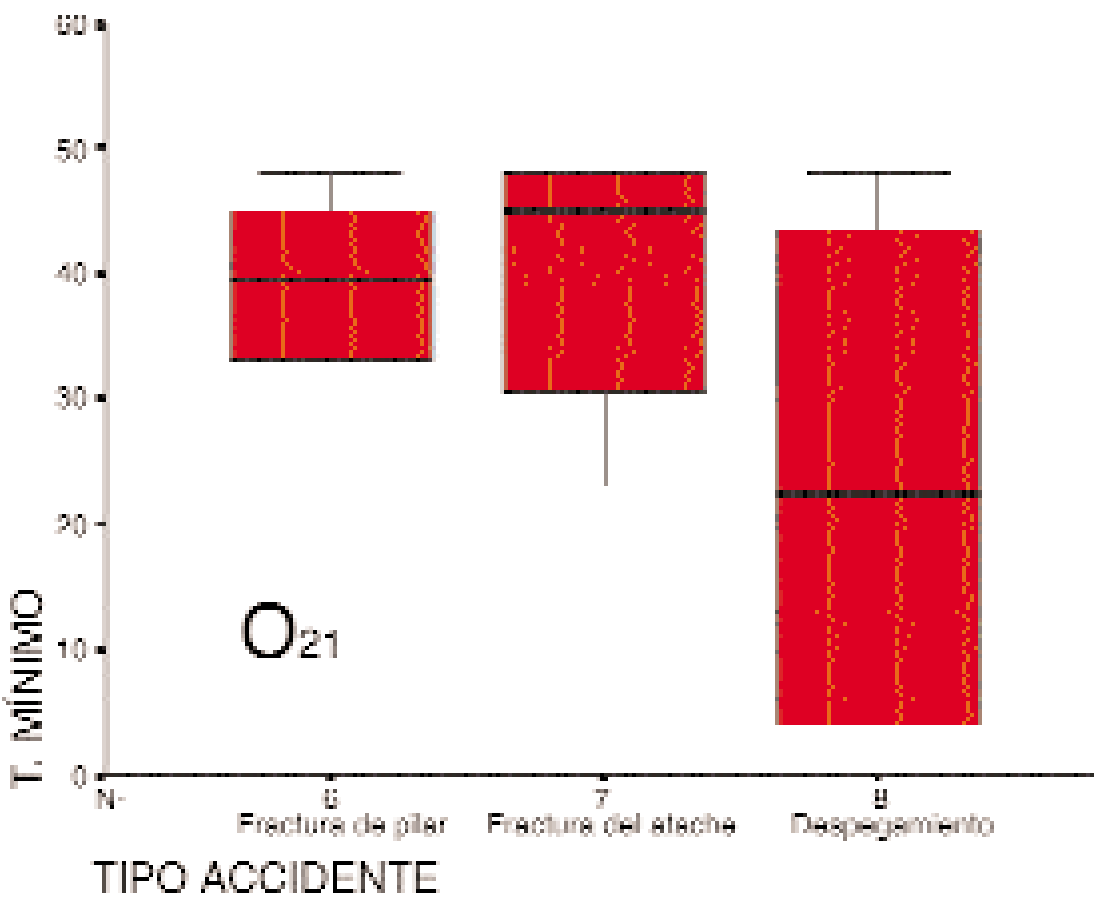

Figura 4. Diagrama de cajas de la distribución del tiempo hasta la primera complicación según tipo de accidente.

ción del tiempo hasta la primera complicación se aprecia en la figura 4.

Los ataches MiniSG presentan el menor número de complicaciones (9\%IC 95\%0 a 18,12). En ningún caso se fracturó el pilar, en un caso se produjo la fractura del atache y en tres el despegamiento de la prótesis fija.

Los ataches MiniDalbo presentaron el mayor número de complicaciones, (17\% IC 95\%-6 a 18,6\%). Sólo el $81 \%$ estaba libre de complicaciones y la fractura del pilar fue la complicación mas frecuente.

Las complicaciones según las variables estudiadas se presentan en la figura 5. Se observa una tendencia a aumentar las complicaciones según disminuye el número de pilares. Las complicaciones son mas frecuentes cuando se trata de un extremo libre (fractura del atache y despegamiento de la prótesis). En la arcada inferior las complicaciones son ligeramente más numerosas. El número de accidentes fue mayor cuando el antagonista fueron dientes naturales.

\section{Estudio analítico}

Observadas las tendencias, realizamos pruebas no paramétricas para conocer si alguno de los factores influía de forma significativa en la presencia de complicaciones. Ninguno de los factores fue significativo. Sin embargo, si pudimos determinar que el factor extremo libre hubiera sido significativo trabajando a un nivel de confianza del $90 \%$ (tabla 2).

Realizamos un análisis de supervivencia de Kaplan-Meier, definiendo la variable supervivencia como el tiempo total en meses desde la colocación del atache hasta la aparición de complica- ciones mecánicas. Y definimos la función de riesgo, tasa de incidencia, como la posibilidad condicionada por unidad de tiempo que tiene cada atache de presentar un accidente, si ha sobrevivido sin complicaciones hasta el instante anterior (fig. 6). La supervivencia media fue de 46 meses (IC 95\% 44,9 a 47,3).

Calculamos la curva de supervivencia según el tipo de accidente (fig. 7). El despegamiento es la complicación mecánica que se presenta los primeros meses. La supervivencia acumulada a los dos años fue del 0,5 cuando estudiamos el despegamiento y casi del 0,9 si nos referimos a fracturas del atache o del pilar. La supervivencia acumulada del despegamiento fue siempre inferior.

Se contrastó la igualdad de las curvas de supervivencia bajo los diferentes niveles de un factor de riesgo, utilizando el test de Breslow y en ningún caso se observó significación estadística (tabla 2).

Se realizó la regresión de Cox y ninguno de los factores fue significativo. Para el tipo de atache el $p$ valor fue de 0,643 y para el extremo libre 0,136 .

\section{Discusión}

El número de complicaciones de nuestro estudio es bajo, tanto de los ataches MiniSG, como los de tipo Roach, que muestran un comportamiento excelente. Ninguno de los factores estudiados, extremo libre, nú-mero de pilares ferulizados, maxilar en que se realiza la rehabilitación y características del antagonista, guarda relación estadística con la presencia de complicaciones. Se observa una tendencia a un compor- 


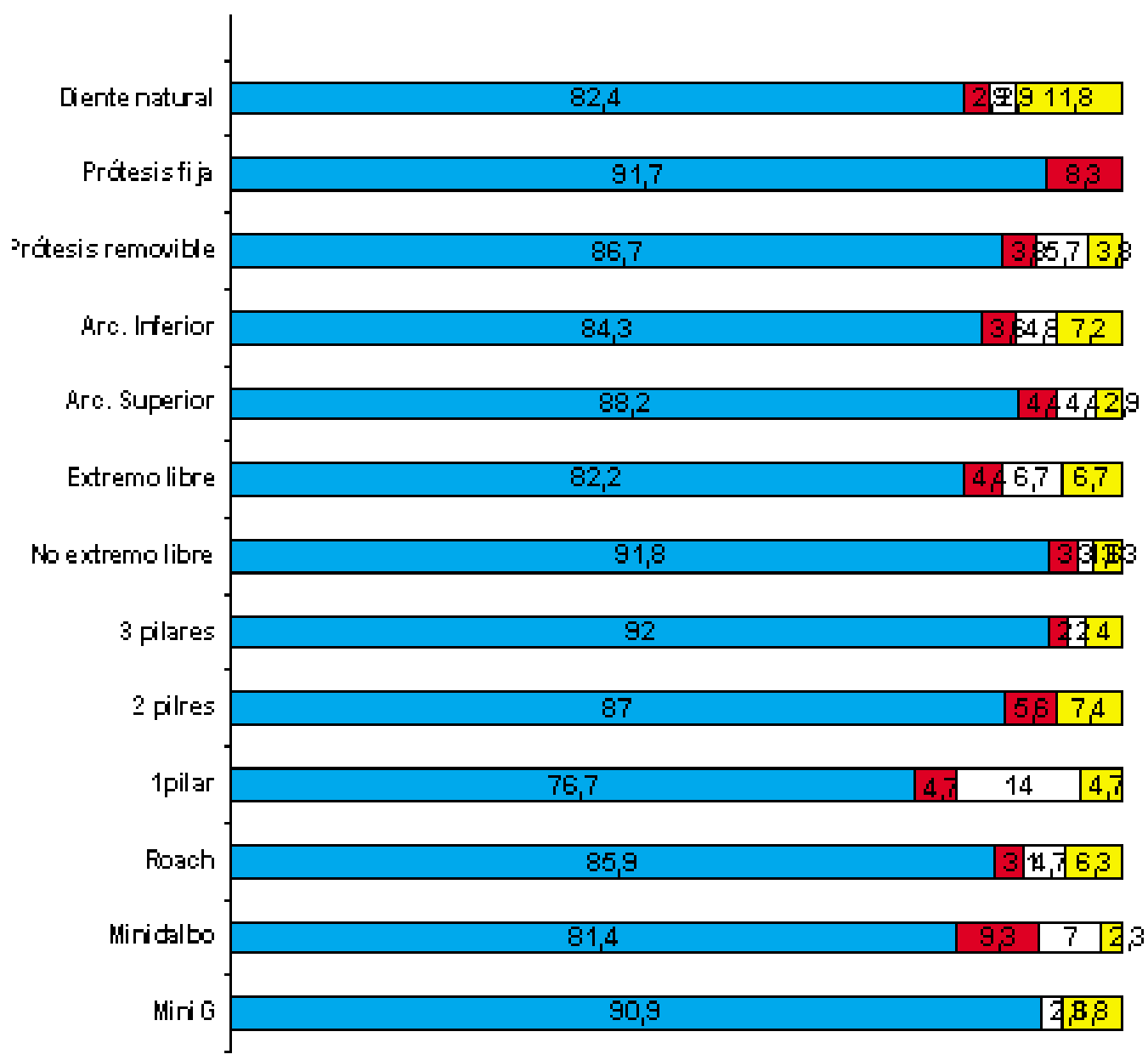

Figura 5. Características de la primera complicación, fractura del pilar, del atache o despegamiento según los aspectos que toman las diferentes variables estudiadas.

tamiento menos eficaz con los ataches MiniDalbo, cuanto menor es el número de pilares ferulizados y en las situaciones de extremo libre.

La función de impacto muestra que el tiempo medio hasta la aparición de la primera complicación es de 32,4 meses. Por este motivo estudios con un periodo de observación menor, aunque muestren resultados notablemente buenos, aportan una información poco relevante ${ }^{11}$.

La prótesis mixta es un tratamiento complejo en que no solo el atache es un elemento clave, sino que la prótesis fija en si misma, constituye un tipo de restauración que puede presentar complicaciones y fracasos. Cheung ${ }^{12}$, utilizando criterios estrictos, en un estudio retrospectivo de 152 coronas unitarias sin ataches con un tiempo medio de servicio en la boca de 34 meses, manifestaba un porcentaje de fracasos del $14 \%$ Estos valores son similares a los hallados por nosotros $(14,7 \%)$ en un periodo de tiempo similar, 4 años, en un tratamiento mucho mas complejo. Las causas pueden ser que establecimos unos criterios de inclusión rigurosos que excluían pacientes parafuncionales y aquellos en que los pilares presentaban enfermedad periodontal. Todos los tratamientos son resultado de una exhaustiva planificación y ejecución minuciosa, habiéndose realizado todos los trabajos en articulador semiajustable y sólo contabilizamos como complicación la fractura del atache, del pilar y el descementado de la prótesis fija.

En ningún caso se ha producido el fracaso del tratamiento, esto es la 


\begin{tabular}{|c|c|c|}
\hline & $\begin{array}{c}\text { Kruskal-Wallis/U de } \\
\text { Mann Whitney }\end{array}$ & $\begin{array}{l}\text { Test de } \\
\text { Breslow }\end{array}$ \\
\hline Tipo de atache & $0,495(\mathrm{KW})$ & 0,590 \\
\hline Número de pilares & $0,130(\mathrm{KW})$ & 0,157 \\
\hline Extremo libre si/no & $0,096(\mathrm{MW})$ & 0,109 \\
\hline Arcada & $0,473(\mathrm{MW})$ & 0,580 \\
\hline Antagonista & $0,695(\mathrm{KW})$ & 0,677 \\
\hline
\end{tabular}

inutilización de la prótesis mixta y la necesidad de plantearnos un tratamiento alternativo.

El despegamiento de la prótesis es la complicación que se presenta con mayor frecuencia en los primeros meses. Del total de complicaciones, el $45 \%$ se deben a despegamiento. En el estudio realizado por Selva ${ }^{13^{*}}$ sobre
101 ataches, el descementado solo supuso el $16 \%$ de las complicaciones, siendo la fractura de los pilares la complicación más frecuente $45 \%$ Besimo $^{14}$ realizó un estudio con 24 ataches extracoronarios y un tiempo de seguimiento de 2,3 años, reportando un porcentaje de descementación de la prótesis fija del 8,3\% En nuestro estudio el porcentaje a los 4 años fue del $6,7 \%$

En nuestro estudio la fractura del pilar es mas frecuente cuando se utilizan ataches MiniDalbo. En los ataches MiniSG nunca se produjo la fractura del pilar. De los problemas hallados por Öwall ${ }^{7 *}$ en una revisión de 57 prótesis con ataches a los 2 años, un $15,8 \%$ se debían a problemas con los pilares; en nuestro caso el $25 \%$ Sin embargo, coincidimos en que el $29,8 \%$ de los problemas se debían al atache, en nuestro caso el $29 \%$ por lo que la responsabilidad del atache en los accidentes fue similar en ambas muestras.

En este estudio de investigación clínica, hemos incluido una serie de variables (extremo libre, número de pilares ferulizados, maxilar en que se realiza la rehabilitación, y características del antagonista) que considerábamos a priori, según la bibliografía
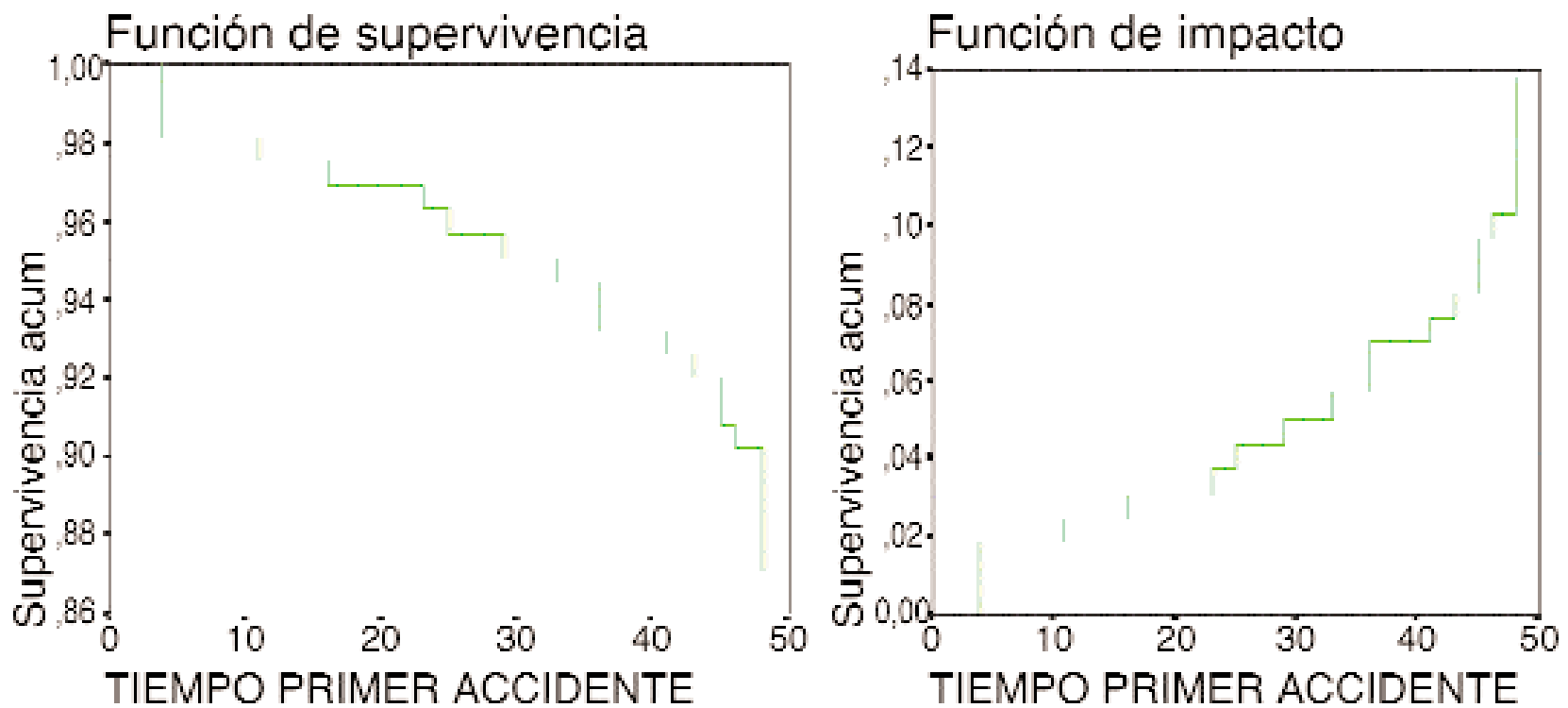

Figura 6. Análisis de supervivencia de Kaplan-Meier. Función de supervivencia y función de impacto. La gráfica solo refleja de 0,86 a 1 ya que en ningún caso la supervivencia acumulada fue inferior a este valor. 


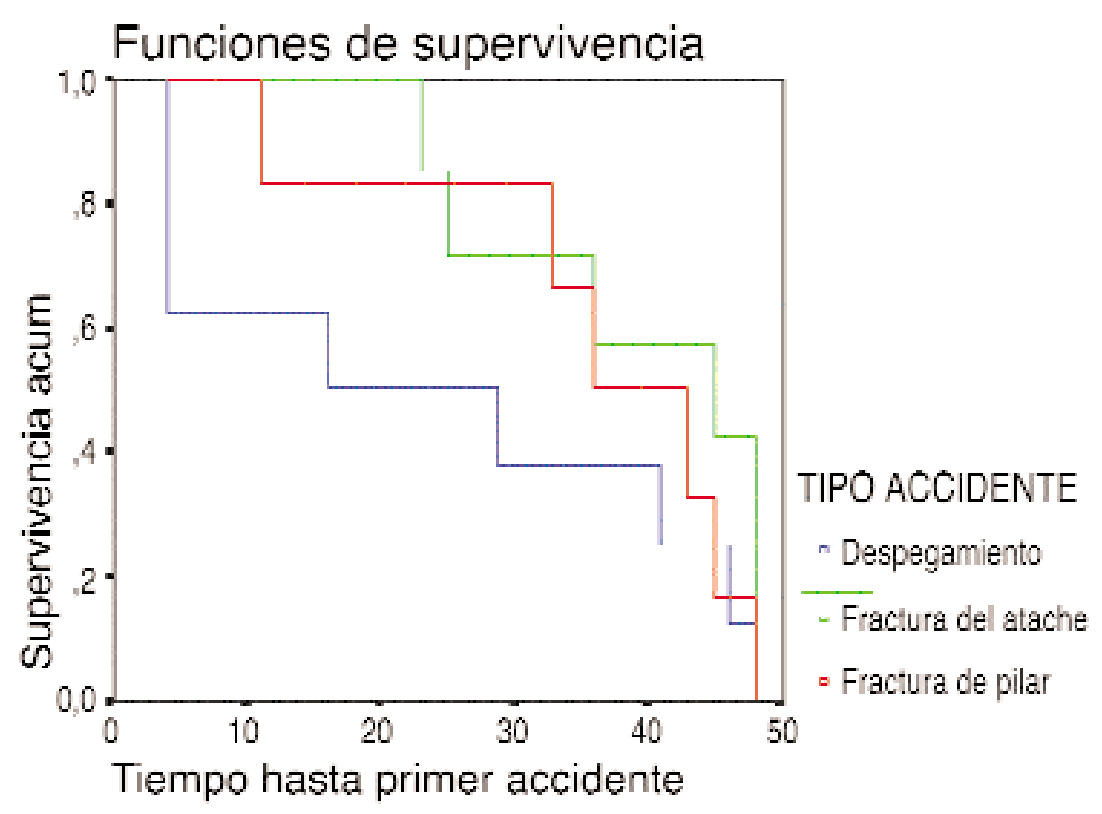

Figura 7. Análisis de supervivencia de Kaplan-Meier. Función de supervivencia según tipo de accidente. La gráfica refleja de 0 a 1 ya que en todos los casos se produjo la complicación.

consultada ${ }^{3-9,15-17}$ podrían influir en el pronóstico de las restauraciones con ataches en prótesis mixtas. En nuestro estudio la aparición de complicaciones no se puede atribuir a ninguna de estas variables sino al azar que conlleva el uso de una muestra para el análisis.

Uno de los factores que más controversia ha suscitado en la literatura científica, es determinar que atache tiene mejor comportamiento clínico: los ataches resilentes o los ataches rígidos. Algunos autores consideran que en todos los casos en los que se pueda crear un equilibrio de fuerzas, se pueden colocar ataches rígidos ${ }^{18,19 *}$.

Studer $^{\beta^{* *}}$ encontró un número importante de fracasos o accidentes en las prótesis parciales removibles cuando se utilizan ataches rígidos. $A$ los 8 años estudio la supervivencia de los ataches Roach a los 4 años fue de $87 \%$ y se presentaron más complicaciones cuando se trata de un atache MiniDalbo.

Los estudios publicados por Owall12 muestran en una muestra de 77 prótesis removibles retenidas con ataches rígidos una tasa de supervivenca del $65 \%$ a los quince años y del 50\%a los veinte años. Nuestro estudio con ataches MiniSG muestra una tasa de supervivencia a los 4 años del 90,9\% El número de complicaciones mecánicas es de 9,1 en ataches MiniSG y de 16,3 en los ataches Roach y MiniDalbo. En el análisis estadístico, a los 48 meses ni las pruebas de Kruskal-Wallis ni de Beslow son significativas. Pero no nos equivoquemos ya que estos datos no indican que el tipo de atache, el extremo libre o el número de pilares ferulizados no influyen en la aparición de complicaciones, lo que afirma es que para el tamaño de muestra presente, no existe evidencia estadística de que las diferencias observadas se deban a factores distintos del mero azar.

\section{Conclusiones}

en las prótesis parciales removibles de extensión distal.

Owall ${ }^{22^{*}}$ en un estudio retrospectivo de 24 prótesis removibles retenidas con ataches tipo Roach, durante un periodo de seguimiento que osciló entre 7 y 282 meses, halló una tasa de éxito del $70 \%$ a los 10 años y del $60 \%$ a los 15 años. Estos resultados son mucho más elevados que los obtenidos por Vermulen ${ }^{23}$ que en sus estudios de 183 prótesis parciales con ataches de precisión incluyendo un 40\% de ataches de bola, halló una supervivencia del 50\%a los 8 años. En nuestro
El atache MiniSG presenta menor número de complicaciones mecánicas que el Roach y que el MiniDalbo, siendo este último en el que más problemas se han constatado, aunque las diferencias no son estadísticamente significativas.

En la muestra estudiada durante 4 años, la aparición de una complicación o el momento en que esta se produce es independiente del tipo de atache y de las demás variables estudiadas. 


\section{Bibliografía recomendada}

Para profundizar en la lectura de este tema, el/los autor/es considera/an interesantes los artículos que aparecen señalados del siguiente modo: *de interés $* *$ de especial interés.

1. Jaroslow B, Gazis D. Multi-attachment approach to partial dentures. Trends Tech Contemp. Dent Lab 1985;2:39-44.

2. Doherty NM. In vitro evaluation of resin-retained extracoronal precision attachments. Int $\mathrm{J}$ Prostodont. 1991;4:63-9.

3. Kratochvil FJ, Tompson WD, Caputo AA. Photoelastic analysis of stress patterns on theeth and bone with attachment retainers for removable partial dentures. J Prosthet Dent 1981;46:21-8.

4. Tsolka P, Altay OT, Preiskel HW. The effect of the Major Connector on Abutment tooth and Denture Base Movement: An In Vitro Study. Int J Prosthodont 1990;3:545-9.

5. Abe M. A clinical survey of removable partial dentures retained by OPA attachments. Observation of male and female. Kokubyo Gakkai Zasshi 1981;48:67-84.

6*. Owall B. Precision attachment-retained removable partial dentures: Part 2. Longterm study of ball attachments. Int J Prosthodont 1995;8:21-8.

Forma parte de una trilogía de artículos sobre prótesis mixta y ataches, que reflejan la experiencia del autor con los diferentes sistemas. El «2» presenta el seguimiento durante 23,5 años de 24 ataches de bola.

7*. Owall B, Jonsson L. Precision attachmentretained removable partial dentures. Part 3 . General practitioner results up to 2 yerars. Int J Prosthodont 1998;11:574-9.

Describe la indicación y la evolución de los ataches colocados en prótesis mixta por un odontólogo general, durante dos años. Son 57 rehabilitaciones, 22 permanecieron libres de complicaciones. 17 tuvieron complicaciones y 9 complicaciones serias. Aquellos con complicaciones tenían retratamiento de conductos y postes.

$8^{* *}$. Studer SP, Mader C, Stahel W, Scharer P. A retrospective study of combined fixed-removable reconstructions with their analysis of failures. J Oral Rehabil 1998;25:513-26.

Es un artículo muy interesante sobre la supervivencia de diferentes tipos de ataches. Es un estudio de muestra grande, en el que se describen y analizan las complicaciones que han presentado las diferentes prótesis mixtas con ataches. Identifica una serie de factores de riesgo: el tipo de atache, la anatomía de la arcada, el extremo libre y el antagonista.

9. Ku YC, Shen YF, Chan CP. Extracoronal resilient attachments in distal-extension removable partial dentures. Quintessence Int 2000; 31:311-7.

10. Shillinburg H. T. Fundamentals of tooth preparation. Chicago:Quintessence Publishing Co,1987.

11. Schwalm CA, Smith DE, Erickson JD. A clinical study of patients 1 to 2 years after placement of removable partial dentures. J Prosthet Dent 1977;38:380-91.

12. Cheung GS. A preliminary investigation into the longevity and causes of failure of single unit extracoronal restorations. J Prosthet Dent 1991;19:160-32.

13*. Selva EJ, Mañes JF, Labaig C, Senent G. Prótesis mixta. Problemática en 101 casos clínicos. Rev Int Prot Estomatol 2001;3:122-125. Es uno de los artículos mas actuales publicados en español y realizados en el estado sobre prótesis mixta, evolución y complicaciones.

14. Besimo C, Gachter M, Jahn M, Hassell T. Clinical perfomance of resin-bonded fixed partial dentures and extracoronal attachments for removable prostheses. J Prosthet Dent 1977; 78:465-71.

15. Zinner ID, Pines MS, Markovits S, Neurohr FG. A stress-relasing intracoronal attachment for extension base removable partial dentures. Gen Den 1998;46(4):398-402.

16. Altay OT, Tsolka P, Preiskel HW. Abutment teeth with extracoronal attachment: the effects of spliting on tooth movement. Int J Prosthodont 1990;3(5):441-8

17. Aydinlik E. Effects of splinting on abutment tooth movement. J Prosthet Dent 1983;49: 477-80.

18. Rantanen T. Investigations of the therapeutic success with dentures retained by precision attachments. Proc Finn Dent Soc 1972;68:73-85.
19*. Preiskel HW. Precision attachment in prosthodontics: The aplications of intracoronalextracoronal attachments. Quintessence Int 1984;12:616-20.

Este autor ha dedicado gran parte de su trabajo a la prótesis mixta, y a los ataches. Ha escrito numerosos artículos y libros de texto que son de gran utilidad para conocer los tipos de ataches, y los aspectos clínicos a tener en cuenta en el diseño; presta especial atención a la importancia del extremo libre. $20 * *$. Burns DR., Ward JE. A review of attachment for removable partial denture desing: part 1. Clasification and selection. Int J Prosthodont 1990;3 (1):98-102.

Analiza las ventajas y desventajas de los distintos tipos de ataches, y las indicaciones y contraindicaciones de los diferentes diseños. Es muy útil para quien desee tener una visión general de los tipos de ataches.

$21 * *$. Burns DR, Ward JE. A review of attachment

for removable partial denture desing: part 2.

Treatment planning and attachment selection. Int J Prosthodont 1990;3(2):169-74.

Describe los diferentes aspectos clínicos y de diseño que se deben de considerar cuando se va ha realiza una prótesis removible con ataches y establece criterios para seleccionar en cada caso el atache mas adecuado. Es un artículo muy interesante y practico para obtener unos conceptos generales y claros sobre las consideraciones ha tener cuando vamos a realizar una prótesis mixta con ataches. Puede ser útil para quien desee iniciarse en la prótesis mixta.

$22 *$. Owall B. Precision attachment retained removable partial denture: 1 . Technical long-term study. Int J Prosthodont 1991;4(3):249-57.

Seguimiento de 53 prótesis mixtas de extensión distal durante 25 años, presenta las tasas de supervivencia y las causas de fracaso. Es el primero de una serie de tres artículos sobre prótesis mixta, en relación con la evolución y las complicaciones.

23. Vermeulen A. Een Decennium Evaluatie van Partiele Prothesen. Een Beschrijvend Klinisch LongitudinalOnderzoek, The Netherlands: Catholic University of Nilmegen, 1984. 